\title{
THE COTYPESET OF A TORSION FREE ABELIAN GROUP OF RANK TWO
}

\author{
C. VINSONHALER AND W. J. WICKLESS
}

\begin{abstract}
The cotypeset (set of types of rank one factors) of a torsion free abelian group of rank two is characterized.
\end{abstract}

Let $G$ be a torsion free abelian group of finite rank, hereafter called simply a "group". The typeset of $G$, \{type $(x) \mid 0 \neq x \in G\}$, has been studied by several authors (e.g. $[3,5,7,8,9,10])$. However, the main problem: When is a set of types the typeset of a group $G$ ?, has not yet been solved, even for groups of rank two. The cotypeset of $G$, introduced in [10], is the set of types of all rank one factors of $G$. This set also seems to be important in the study of torsion free groups (see e.g. [1, 2, 11, 12, 13]). It therefore seems of interest to characterize those sets of types which are the cotypeset of a group $G$. In this paper we give necessary and sufficient conditions for a set of types to be the cotypeset of a rank two group.

Familiarity is assumed with the notions of type and characteristic (height vector) - see [6]. In particular, the inner type, IT, and outer type OT, of Warfield are used [13]. The symbols $\vee$ and $\wedge$ are used to denote the sup and inf, respectively, of collections of characteristics. If $t$ is a characteristic, $t^{p}$ denotes the value of $t$ at the prime $p$. If $\tau$ is a type, $\tau^{p}$ is called finite (infinite), if $t^{p}$ is finite (infinite) for some characteristic $t \in \tau$. If $\tau_{1}$ and $\tau_{2}$ are types with $\tau_{1} \leqslant \tau_{2}$, then $\tau_{2}-\tau_{1}$ is the type of $t_{2}-t_{1}$, where $t_{1} \in \tau_{1}, t_{2} \in \tau_{2}$ are characteristics chosen so that $t_{1} \leqslant t_{2}$ (note: $\infty-\infty=0$ ). The type of $Z$ is denoted by 0 . Finally, we call two types, $\tau_{1}$ and $\tau_{2}$, equivalent on a subset $P$ of the primes $\left(\tau_{1} \sim \tau_{2}\right.$ on $\left.P\right)$ if there exist characteristics $t_{1} \in \tau_{1}, t_{2} \in \tau_{2}$ such that $t P=t t_{2}$ for all $p \in P$.

The characterization of rank two groups by Beaumont-Wisner [4] is employed repeatedly. As usual, $h_{G}^{P}(x)$ denotes the $p$-height of an element $x$ in a group $G$, and $\operatorname{type}_{G}(x)$ is the type of $x$ in $G$. We use \langle\rangle$\left(\langle\rangle_{*}\right)$ to denote the subgroup (pure subgroup) generated by a set of elements.

We begin with a simple lemma.

LEMMA 1. Let $S=\left\{\sigma_{i}\right\}_{i \in I}$ be a set of types and $\sigma_{0}$ a type such that $\sigma_{0}=\sigma_{i} \vee \sigma_{j}$ for all $i \neq j$ in $I$. Then

(a) $\sigma_{0}-\sigma_{i} \leqslant \sigma_{j}$ for all $i \neq j$ in $I$.

(b) $\left(\sigma_{0}-\sigma_{i}\right) \wedge\left(\sigma_{0}-\sigma_{j}\right)=\mathbf{0}$ for all $i \neq j$ in $I$.

Received by the editors January 15, 1981 and, in revised form, May 27, 1981.

1980 Mathematics Subject Classification. Primary 20K15. 
Proof. Obvious.

A special case of the main theorem is proved first.

TheOREM 1. Let $S=\left\{\sigma_{i}\right\}$ be a finite or countable set of types. Then $S$ is the cotypeset of a group $G$ of rank two with $\operatorname{IT}(G)=0$ if and only if there exists a type $\sigma_{0}$ such that:

(1) $\sigma_{0}=\sigma_{i} \vee \sigma_{j}$ for all $i \neq j$, and if $S$ is finite, $\sigma_{0}=\sigma_{i}$ for some $i$.

(2) $\sigma_{j}-\left(\sigma_{0}-\sigma_{i}\right)=\sigma_{i}-\left(\sigma_{0}-\sigma_{j}\right)$ for all $i \neq j$.

Proof. I. Necessity. Suppose $S$ is the cotypeset of a rank two group $G$ with $\operatorname{IT}(G)=0$. Let $\sigma_{0}=\operatorname{OT}(G)$, the outer type of $G$. Then the first claim of condition (1) is immediate. For all $i$, choose $x_{i} \in G$ such that $G /\left\langle x_{i}\right\rangle_{*}$ has type $\sigma_{i}$. If $S=\left\{\sigma_{1}, \ldots, \sigma_{n}\right\}$ is finite, choose $0 \neq x \in G$ such that $\langle x\rangle_{*} \neq\left\langle x_{i}\right\rangle_{*}, i=1, \ldots, n$. Let $\sigma=$ type $G /\langle x\rangle_{*}$. Then $\sigma \in S$ and $\sigma \vee \sigma_{i}=\sigma_{0}$ for all $i$. Thus, $\sigma=\sigma_{0}$.

To establish the remaining claim, for each $i$ define $\tau_{i}=\sigma_{0}-\sigma_{i}$ and let $\tau_{i}^{\prime}=$ type $x_{i}$. It is well known that since $G$ is rank two of inner type $0, \tau_{i}^{\prime}+\sigma_{i}=\sigma_{0}$. It follows that we can choose characteristics $t_{i} \in \tau_{i}, t_{i}^{\prime} \in \tau_{i}^{\prime}$ with $t_{i}^{p}=\left(t_{i}^{\prime}\right)^{p}$ for all primes $p$ except possibly those for which $\sigma_{i}^{p}=\sigma_{0}^{p}=\infty$. At all such $p$ we can set $t_{i}^{p}=0$, although $\left(t_{i}^{\prime}\right)^{p}$ can be any nonnegative integer. Thus $t_{i} \leqslant t_{i}^{\prime}$, but equality need not hold.

If $i \neq j$, let $X_{i}=G /\left\langle x_{i}\right\rangle_{*}, X_{j}=G /\left\langle x_{j}\right\rangle_{*}$. Then $X_{i}$ and $X_{j}$ are rank one groups such that $X_{i} \supseteq\left\langle x_{j}\right\rangle_{*}, X_{j} \supseteq\left\langle x_{i}\right\rangle_{*}$ under the natural embeddings. It is well known (see e.g. [4]) that $X_{j} /\left\langle x_{i}\right\rangle_{*} \cong X_{i} /\left\langle x_{j}\right\rangle_{*}$. The isomorphism implies that we can choose characteristics $s_{i} \in \sigma_{i}, s_{j} \in \sigma_{j}$ with $s_{j}-t_{i}^{\prime}=s_{i}-t_{j}^{\prime}$. Thus, in view of our earlier remarks, if $p$ is a prime with $s_{i}^{p}$ and $s_{j}^{p}$ finite, then $t_{i}^{p}=\left(t_{i}^{\prime}\right)^{p}, t_{j}^{p}=\left(t_{j}^{\prime}\right)^{p}$, and $s_{j}^{p}-t_{i}^{p}=s_{i}^{p}-t_{j}^{p}$. If $s_{i}^{p}=s_{j}^{p}=\infty$, then $t_{i}^{p}=t_{j}^{p}=0$, so again $s_{j}^{p}-t_{i}^{p}=s_{i}^{p}-t_{j}^{p}$. Let $p$ be a prime for which $s_{i}^{p}=\infty, s_{j}^{p}<\infty$. Then $\infty=s_{0}^{p}=s_{j}^{p}+\left(t_{j}^{\prime}\right)^{p}$, so $\left(t_{j}^{\prime}\right)^{p}=\infty$. Thus, $0=s_{i}^{p}-\left(t_{j}^{\prime}\right)^{p}=s_{j}^{p}-\left(t_{i}^{\prime}\right)^{p}$. Moreover, $\tau_{i}^{\prime} \wedge \tau_{j}^{\prime}=0$, so $\tau_{i}^{\prime} \sim 0$ on the set of all primes $p$ where $\left(\tau_{j}^{\prime}\right)^{p}=\infty$.

Since $\tau_{i} \leqslant \tau_{i}^{\prime}$ the same claim holds for $\tau_{i}$. Thus, the equation $s_{j}^{p}-t_{i}^{p}=s_{i}^{p}-t_{j}^{p}$ holds for almost all $p$ where $s_{i}^{p}=\infty, s_{j}^{p}<\infty$, and where the equality fails both sides are finite. By symmetry the same statement is true for the set of primes where $s_{i}^{p}<\infty, s_{j}^{p}=\infty$. This establishes condition (2).

II. Sufficiency.

A. Choosing characteristics. Let $S=\left\{\sigma_{i}\right\}$ be a finite or countable set of types satisfying conditions (1) and (2). Define $\tau_{i}=\sigma_{0}-\sigma_{i}$ as before. We show $\left\{\tau_{i}\right\}$ is relatively disjoint, that is, there exists a set of characteristics $t_{i} \in \tau_{i}$ such that $t_{i}^{p} \wedge t_{j}^{p}=0$ for all primes $p$ and $i \neq j$. Suppose $t_{1} \in \tau_{1}, \ldots, t_{n} \in \tau_{n}$ have been chosen such that:

(i) for all $p$ and $1 \leqslant i<j \leqslant n, t_{i}^{p} \wedge t_{j}^{p}=0$,

(ii) $t_{i}^{p}=0$ if $\tau_{k}^{p}=\infty$ for any $k \in Z^{+}, k \neq i$.

Condition (ii) is possible since if $\tau_{k}^{p}=\infty$ then $\sigma_{0}^{p}$ and $\sigma_{i}^{p}$ must both be infinite by Lemma 1(a) and Condition (1) of the theorem. Thus $\tau_{i}=\sigma_{0}-\sigma_{i}$ has a characteristic which is 0 at all such $p$. Choose $t_{n+1}^{\prime} \in \tau_{n+1}$. Note that if $\left(t_{n+1}^{\prime}\right)^{p}=\infty$ for some $p$ then $t_{i}^{p}=0$ for $1 \leqslant i \leqslant n$. Furthermore, $\Pi_{i}=\left\{p \mid 0<\left(t_{n+1}^{\prime}\right)^{p} \wedge t_{i}^{p}<\infty\right\}$ is finite 
for each $i$ by Lemma 1(b). Define $t_{n+1}^{p}=0$ if $p \in \cup_{i=1}^{n} \Pi_{i}$ or if $\tau_{k}^{p}=\infty$ for some $k \in Z^{+}, k \neq n+1$; and $t_{n+1}^{p}=\left(t_{n+1}^{\prime}\right)^{p}$ otherwise. Then $t_{n+1} \in \tau_{n+1}$ and $t_{1}, \ldots, t_{n+1}$ satisfy (i) and (ii). By induction, a set $\left\{t_{i}\right\}$ of characteristics can be chosen with $t_{i} \in \tau_{i}$ and $t_{i}^{p} \wedge t_{j}^{p}=0$ for all $p, i \neq j$.

B. Ito's construction [7]. Given a relatively disjoint set of types $\left\{\tau_{i}\right\}$, Ito constructs a rank two group $G$ with typeset $G=\left\{\tau_{i}\right\}$ as follows. Let $t_{i} \in \tau_{i}$ be a set of relatively disjoint characteristics. For each $i$, choose a rank one group $G_{i}$ of type $\tau_{i}$, and $x_{i} \in G_{i}$ whose characteristic is exactly $t_{i}$. Let $\left\{\left(a_{i}, b_{i}\right) \mid 1 \leqslant i<\infty\right\}$ be the set of all coprime pairs of integers with $b_{i} \geqslant 0$, numbered so that $\operatorname{Max}\left\{\left|a_{i}\right|, b_{i}\right\} \leqslant i$. Then if $H=\bigoplus_{i=3}^{\infty}\left\langle a_{i} x_{1}+b_{i} x_{2}-x_{i}\right\rangle$ and $G=\bigoplus_{i=1}^{\infty} G_{i} / H$, Ito shows that $G$ is a torsion free rank two group, and that the element $g_{i}=a_{i} \bar{x}_{1}+b_{i} \bar{x}_{2}=a_{i}\left(x_{1}+H\right)+$ $b_{i}\left(x_{2}+H\right)$ has type $\tau_{i}$ in $G$.

Two additional facts about Ito's construction can be noted. First, he considers only the case when $\left\{\tau_{i}\right\}$ is infinite, the finite case having been settled in [3]. However, his construction also works if $\left\{\tau_{i}\right\}$ is finite. In this case, all but a finite number of the elements $\left\{g_{i}\right\}$ are assigned the type 0 , which is necessarily in element of $\left\{\tau_{i}\right\}$. Second, an easy computation shows that the outer type of the rank two group constructed, OT $(G)$, is the type represented by the characteristic $V_{i} t_{i}$.

Henceforth we assume $S=\left\{\sigma_{i}\right\}$ is a set of types satisfying conditions (1) and (2) of the theorem, $\tau_{i}=\sigma_{0}-\sigma_{i}$ and $\left\{t_{i}\right\}$ is a relatively disjoint set of characteristics with $t_{i} \in \tau_{i}$. Let $G_{1}$ be the group constructed by Ito's method with typeset $G_{1}=\left\{\tau_{i}\right\}$, and let $\sigma=\mathrm{OT}\left(G_{1}\right)$ with representative $s=V_{i} t_{i}$.

C. Changing OT $\left(G_{1}\right)$. In general $\sigma \leqslant \sigma_{0}$, since each $\tau_{i} \leqslant \sigma_{0}$. Choose $s_{0} \in \sigma_{0}$ with $s^{p} \leqslant s_{0}^{p}$ for all primes $p$. Let $P_{1}=\left\{p \mid 0<s_{0}^{p}-s^{p}<\infty, P_{2}=\left\{p \mid s^{p}<\infty, s_{0}^{p}=\infty\right\}\right.$. In this section we construct a rank two group $G \geqslant G_{1}$ such that:

(i) $\operatorname{IT}(G)=\mathbf{0}$,

(ii) $\mathrm{OT}(G)=\sigma_{0}$,

(iii) for all $g_{i}$, type ${ }_{G}\left(g_{i}\right) \sim$ type $_{G_{1}}\left(g_{i}\right)$ on the complement of $P_{2}\left(\right.$ denoted $\left.P_{2}^{c}\right)$,

(iv) for all $g_{i}$ and $p \in P_{2}, h_{G}^{p}\left(g_{i}\right)<\infty$.

First note that if $\left|P_{1}\right|<\infty$ we already have $\sigma \sim \sigma_{0}$ on $P_{2}^{c}$. In this case we leave the $p$ height of all $g_{i}$ unchanged for $p \in P_{2}^{c}$. Otherwise, index the primes in $P_{1}$ in their natural order, say $P_{1}=\left\{p_{j}\right\}$. It is easy to see that we can select a subsequence $\left(a_{j}, b_{j}\right)$, with all terms distinct, from our original sequence $\left(a_{i}, b_{i}\right)$ of coprime pairs of integers, such that: $\lim _{j \rightarrow \infty} j / p_{j}=0$ and if $t_{i}^{p_{j}}>0$ then

$$
\operatorname{det}\left(\begin{array}{ll}
a_{j} & b_{j} \\
a_{i} & b_{i}
\end{array}\right) \equiv 0 \quad\left(\bmod p_{j}\right) .
$$

(Note that for each $p_{j} \in P_{1}$ there is at most one $t_{i}$ with $t_{i}^{p_{j}}>0$.) Let

$$
G_{2}=\left\langle G_{1},\left\{g_{j} / p_{j}^{n_{j}} \mid p_{j} \in P_{1}\right\}\right\rangle,
$$

where $n_{j}=s_{0}^{p_{j}}-h_{G_{1}}^{p_{j}}\left(g_{j}\right)$. It is apparent that, for $p \notin P_{1}$, the $p$ height of any $g_{i}$ is unchanged in going from $G_{1}$ to $G_{2}$. Furthermore, if $h_{G_{2}}^{p_{j}}\left(g_{i}\right)>0$ then

$$
\operatorname{det}\left(\begin{array}{ll}
a_{i} & b_{i} \\
a_{j} & b_{j}
\end{array}\right) \equiv 0 \bmod \left(p_{j}\right)
$$


[5, part I]. But $\left|a_{i} b_{j}-a_{j} b_{i}\right| \leqslant 2 i j$ and $\lim _{j \rightarrow \infty} j / p_{j}=0$. Thus, we can have $h_{G_{2}}^{p_{j}}\left(g_{i}\right)>0$ for only finitely many $p_{j} \in P_{1}$, and type $G_{G_{2}}\left(g_{i}\right)=\operatorname{type}_{G_{1}}\left(g_{i}\right)$ for all $g_{i}$. Since each element on $G_{2}$ is rationally dependent on some $g_{i}$, it follows that typeset $G_{2}=$ typeset $G_{1}$. Finally, it is easy to see that the outer type of $G_{1}$ has been increased so that $\mathrm{OT}\left(G_{2}\right) \sim \sigma_{0}$ on $P_{2}^{c}$.

We now consider the set of primes $P_{2}$, on which the outer type must be made infinite. Let $X_{1}=G_{2} /\left\langle\bar{x}_{1}\right\rangle_{*}$ and $X_{2}=G_{2} /\left\langle\bar{x}_{2}\right\rangle_{*}$. As in I (Necessity), $X_{1} /\left\langle\bar{x}_{2}\right\rangle_{*}$ $\stackrel{\theta}{\cong} X_{2} /\left\langle\bar{x}_{1}\right\rangle_{*}$, where $\theta$ is uniquely determined by $G_{2}$. Let $R$ be the subring of $Q$ generated by $\left\{1 / p \mid p \in P_{2}\right\}$. By definition of $P_{2}$, none of the rank one groups $X_{1}$, $\left\langle\bar{x}_{1}\right\rangle_{*}, X_{2},\left\langle\bar{x}_{2}\right\rangle_{*}$ is $p$-divisible for $p \in P_{2}$. Thus $R \otimes_{Z} X_{1} /\left\langle\bar{x}_{2}\right\rangle_{*} \stackrel{\theta^{\prime}}{=} R \otimes_{Z} X_{2} /\left\langle\bar{x}_{1}\right\rangle_{*}$ and the isomorphism $\theta^{\prime}$ can be chosen so that

(*) $\theta^{\prime}$ is an irrational $p$-adic unit on each $p$ component such that $p \in P_{2}$.

$(* *)$ The natural diagram commutes:

$$
\begin{array}{ccc}
X_{1} /\left\langle\bar{x}_{2}\right\rangle_{*} & \stackrel{\theta}{\cong} & X_{2} /\left\langle\bar{x}_{1}\right\rangle_{*} \\
\downarrow & & \downarrow \\
R \otimes_{Z} X_{1} /\left\langle\bar{x}_{2}\right\rangle_{*} & \stackrel{\theta^{\prime}}{\cong} & R \otimes_{Z} X_{2} /\left\langle\bar{x}_{1}\right\rangle_{*}
\end{array}
$$

Let

$$
G=\left\{(\alpha, \beta) \mid \alpha \in R \otimes X_{1}, \beta \in R \otimes X_{2} \text { and } \theta^{\prime}\left(\alpha+\left\langle\bar{x}_{2}\right\rangle_{*}\right)=\beta+\left\langle\bar{x}_{1}\right\rangle_{*}\right\} .
$$

The following facts about $G$ are easily verified:

(a) The map $\beta \bar{x}_{1}+\alpha \bar{x}_{2} \rightarrow(\alpha, \beta)$ gives a natural embedding of $G_{2}$ into $G$ (use $(* *))$.

(b) Under this embedding $h_{G_{2}}^{P}(x)=h_{G}^{P}(x)$ for all $x \in G_{2}$ and $p \notin P_{2}$.

(c) $h_{G}^{P}(x)<\infty$ for all $0 \neq x \in G$ and $p \in P_{2}$ (use (*)).

(d) OT $(G)$ is infinite at all primes $p \in P_{2}$.

(e) $\mathrm{OT}(G) \sim \mathrm{OT}\left(G_{2}\right)$ on $P_{2}^{c}$.

(f) $\operatorname{IT}(G)=0$. (The embedding maps $\left\langle\bar{x}_{1}\right\rangle_{*}$ and $\left\langle\bar{x}_{2}\right\rangle_{*}$ onto distinct pure subgroups of $G$.)

In view of (a)-(f), $G$ is a group satisfying conditions (i)-(iv) stated at the beginning of this section.

D. The cotypeset of $G$. In this section we complete the proof of Theorem 1 by showing that the cotypeset of $G$ is exactly $S$. By the results of the previous section, $\mathrm{OT}(G)=\sigma_{0}$ and the typeset of $G$ is $\left\{\tau_{i}^{\prime}\right\}$ where $\tau_{i}^{\prime} \sim \tau_{i}=\sigma_{0}-\sigma_{i}$ on $P_{2}^{c}$ and $\tau_{i}^{\prime}<\infty$ at all primes $p \in P_{2}$. For each $i$, let $g_{i}$ be an element of $G$ with type $\tau_{i}^{\prime}$. Then, type $G /\left\langle g_{i}\right\rangle_{*}=\sigma_{i}^{\prime}$ where $\sigma_{i}^{\prime}+\tau_{i}^{\prime}=\sigma_{0}$. Moreover, for all $i \neq j, \sigma_{i}^{\prime}-\tau_{j}^{\prime}=\sigma_{j}^{\prime}-\tau_{i}^{\prime}$ and $\tau_{i}^{\prime} \wedge \tau_{j}^{\prime}=0$. We will show $\sigma_{i}^{\prime}=\sigma_{i}$ for all $i$. The proof is divided into four cases.

Case I. $p \in P_{2}$. For $p \in P_{2}$, $\sigma^{p}$ is infinite while $\tau_{i}^{p}$ and $\tau_{i}^{p}$ are both finite. Thus both $\sigma_{i}^{p}$ and $\sigma_{i}^{\prime p}$ are infinite on $P_{2}$.

Case II. $\sigma_{0}^{p}$ is finite. Let $P_{3}=\left\{p \mid \sigma_{0}^{p}<\infty\right\}$. Then $\tau_{i} \sim \tau_{i}^{\prime}$ on $P_{3}$ since $P_{3} \subseteq P_{2}^{c}$. Since $\sigma_{0}=\sigma_{i}+\tau_{i}=\sigma_{i}^{\prime}+\tau_{i}^{\prime}$, then $\sigma_{i} \sim \sigma_{i}^{\prime}$ on $P_{3}$. 
Case III. $\sigma_{0}^{p}=\infty, p \notin P_{2}, \sigma_{i}^{p}=\infty$. At these primes, $\tau_{i}^{\prime} \sim \tau_{i} \sim \mathbf{0}$, and hence $\sigma_{i}^{\prime}$ is infinite and equal to $\sigma_{i}$.

Case IV. $\sigma_{0}^{P}=\infty, \sigma_{i}^{p}<\infty, p \notin P_{2}$.

For each fixed $i$, this case can occur for only a finite number of primes $p$. This follows from the equations, $\sigma_{i}-\tau_{j}=\sigma_{j}-\tau_{i}$ (Condition (2)) and $\tau_{i} \wedge \tau_{j}=\mathbf{0}$ (Lemma 1(b)). Since $\tau_{i}$ infinite implies $\tau_{i}^{\prime}$ infinite, then $\sigma_{i}^{\prime}$ is finite and hence equivalent to $\sigma_{i}$ on this finite set of primes. Cases I-IV imply $\sigma_{i}=\sigma_{i}^{\prime}$ for all $i$.

REMARK 1. In the proof of sufficiency (II) we have tacitly assumed $S=\left\{\sigma_{i}\right\}$ contains more than one element. If $S=\left\{\sigma_{0}\right\}$ is a set containing only one type, it is easy to construct a rank two group $G$, homogeneous of type 0 , whose cotypeset is $S$.

REMARK 2. Theorem 1 would be a trivial consequence of $\$ \$ 5$ and 6 of [10] if the results therein were true.

However, as the following example shows, the results in [10] are incorrect.

EXAMPLE. The following adaptation of an example of Dubois shows that conditions (1)-(4) of Proposition 4 of [10] are not sufficient to guarantee that a set of type-cotype pairs $\left\{\left(\tau_{i}, \sigma_{i}\right) \mid 1 \leqslant i<\infty\right\}$ is the type-cotype set of a rank two group. Let $P$ be the set of primes in $Z$ and write $P=\cup_{i=1}^{\infty} P_{i}$ as a disjoint union of countable sets $P_{i}$. Let $t_{1}$ be the characteristic defined by $t p=1$ for $p \in P_{1}, t P=0$ otherwise. For $i>1$ define $t_{i}^{p}=1$ for $p \in P_{i}, t_{i}^{p}=\infty$ if $p$ is the $i$ th prime in $P_{j}$ for some $j<i, t_{i}^{p}=0$ otherwise. Let $\tau_{i}$ be the type of $t_{i}$ for $1 \leqslant i<\infty$, and let $\sigma_{0}=$ type $Q, \sigma_{i}=\sigma_{0}-\tau_{i}$ for $1 \leqslant i<\infty$. It is easy to check that the set $\left\{\left(\tau_{i}, \sigma_{i}\right) \mid 1 \leqslant\right.$ $i<\infty\}$ satisfies (1)-(4) of Proposition 4 of [10]. However, as shown by Dubois, $\left\{\tau_{i} \mid 1 \leqslant i<\infty\right\}$ is not the type set of any rank two group.

A final lemma precedes the Main Theorem.

LEMMa 2. Let $S=\left\{\sigma_{i}\right\}$ be a finite or countable set of types. Then $S$ is the cotypeset of a rank two group $G$ if and only if there exists a type $\tau_{0}$ such that (1) $\tau_{0} \leqslant \sigma_{i}$ for all $i$, (2) $\left\{\sigma_{i}-\tau_{0}\right\}$ is the cotypeset of a rank two group of inner type $\mathbf{0}$. (That is, $\left\{\sigma_{i}-\tau_{0}\right\}$ satisfies the conditions of Theorem 1.)

Proof. Suppose $S=\left\{\sigma_{i}\right\}$ is the cotypeset of a rank two group $G$. Let $\tau_{0}=\operatorname{IT}(G)$. Clearly, $\tau_{0} \leqslant \sigma_{i}$ for all $i$. As in [10], $G$ can be written $G=U \otimes G_{0}$, where $U$ is a rank one group of type $\tau_{0}$ and $G_{0}$ is a rank two group of inner type 0 . Furthermore, $G_{0}$ can be chosen so that OT $\left(G_{0}\right) \sim 0$ on the set of primes $p$ where $\tau_{0}^{P}$ is infinite. Let $K$ be a pure rank one subgroup of $G$. Then there is an obvious isomorphism: $G / K \cong U \otimes G_{0} / U \otimes K_{0} \cong U \otimes\left(G_{0} / K_{0}\right)$, where $K_{0}$ is a pure rank one subgroup of $G_{0}$. Conversely, if $K_{0}$ is a pure rank one subgroup of $G_{0}$, then $K=U \otimes K_{0}$ is a pure rank one subgroup of $G=U \otimes G_{0}$, and the same isomorphism holds. Furthermore, type $G / K=\tau_{0}+$ type $G_{0} / K_{0}$, and therefore, type $G_{0} / K_{0}=$ type $G / K-\tau_{0}$, in view of our choice of $G_{0}$. It follows that cotypeset $G_{0}$ is exactly $\left\{\sigma_{i}-\tau_{0}\right\}$.

Conversely, suppose there exists a type $\tau_{0}$ such that $\left\{\sigma_{i}-\tau_{0}\right\}$ is the cotypeset of a rank two group $G_{0}$ of inner type 0 . If $\tau_{0}<$ type $Q$ let $U$ be a rank one group of type $\tau_{0}$. Then $G=U \otimes G_{0}$ is a (reduced) group $G$ with cotypeset $\left\{\sigma_{i}\right\}$. If $\tau_{0}=$ type $Q$, then $S=\{$ type $Q\}$ by (1) and (2) holds trivially. As mentioned previously, in this case it is not difficult to construct the desired rank two group $G$. 
THEOREM 2. Let $S=\left\{\sigma_{i}\right\}$ be a finite or countable set of types. Then $S$ is the cotypeset of a rank two group $G$ if and only if there exists a type $\sigma_{0}$ such that $\sigma_{i} \vee \sigma_{j}=\sigma_{0}$ if $i \neq j$, subject to the additional requirement that $\sigma_{0} \in S$ if $|S|<\infty$.

PROoF. The condition is clearly necessary. To prove sufficiency, we produce a type $\tau_{0}$ such that $\left\{\sigma_{i}-\tau_{0}\right\}$ satisfies the conditions of Theorem 1 and apply Lemma 2.

In view of our earlier remarks, we may assume $|S|>1$. Choose $s_{0} \in \sigma_{0}, s_{1} \in \sigma_{1}$ with $s_{1}^{p} \leqslant s_{0}^{p}$ for all $p$. If $\left\{s_{1}, \ldots, s_{n}\right\}$ have been chosen, choose $s_{n+1} \in \sigma_{n+1}$ with $s_{n+1}^{p} \leqslant s_{0}^{p}$, for all $p$, and with $s_{n+1}^{p} \vee s_{j}^{p}=s_{0}^{p}$, for all $p$ and $1 \leqslant j \leqslant n$. This choice is possible since $\sigma_{n+1} \vee \sigma_{j}=\sigma_{0}, 1 \leqslant j \leqslant n$. Thus, by induction, we have a set of characteristics $s_{i} \in \sigma_{i}$ with $s_{i} \vee s_{j}=s_{0}$. Note that for all $p$ there is at most one $s_{i}$ with $s_{i}^{p}<s_{0}^{p}$. Let $t_{0}$ be the characteristic defined by $t_{0}^{p}=\wedge s_{i}^{p}$ and let $\tau_{0}$ be the type represented by $t_{0}$. It is straightforward to verify that $\left\{\sigma_{i}^{\prime}=\sigma_{i}-\tau_{0} \mid 1 \leqslant i<\infty\right\}$ satisfies conditions (1) and (2) of Theorem 1 with respect to the type $\sigma_{0}^{\prime}=\sigma_{0}-\tau_{0}$. The Theorem now follows from Lemma 2.

The inner type $\tau_{0}$ is not, in general, uniquely determined by the cotypeset $S$, due to the different possible choices for the characteristics $s_{i} \in \sigma_{i}$. A characterization of the possible inner types for a group $G$ with cotypeset $S$ is given by

Theorem 3. Let $S=\left\{\sigma_{i}\right\}$ and $\tau_{0}$ be as in Theorem 2. Let $S_{i}=\left\{p \mid s_{i}^{p}=t_{0}^{p}<\infty\right.$ and $\left.s_{0}^{p}=\infty\right\}$. Then a type $\tau$ with characteristic $t$ is the inner type of a rank 2 group $G$ with cotypeset $S$ if and only if for all $i, \tau \leqslant \sigma_{i}$ and $\left\{p \mid t^{p} \neq t_{0}^{p}\right\} \cap S_{i}$ is finite.

Proof. If $\tau$ is a type satisfying these hypotheses, it is easy to show that $\left\{\sigma_{i}-\tau\right\}$ satisfies the conditions of Theorem 1. Thus, as in the proof of Lemma 2, there is a rank 2 group $G$ of inner type $\tau$ and cotypeset $S$.

Conversely, if $\tau$ is the inner type of a rank 2 group with cotypeset $S$, then $\left\{\sigma_{i}-\tau\right\}$ satisfies the conditions of Theorem 1 .

In particular, if $i \neq j$, then

$$
\left(\sigma_{j}-\tau\right)-\left[\left(\sigma_{0}-\tau\right)-\left(\sigma_{i}-\tau\right)\right]=\left(\sigma_{i}-\tau\right)-\left[\left(\sigma_{0}-\tau\right)-\left(\sigma_{j}-\tau\right)\right] .
$$

Let $P_{i}=S_{i} \cap\left\{p \mid t^{p} \neq t_{0}^{p}\right\}$. Then, on this set of primes the left hand side of $(\dagger)$ is $\sim 0$, while the right-hand side is $\sim\left(\sigma_{i}-\tau\right)$. Since, on $P_{i}, \sigma_{i} \sim \tau_{0}$, we have $\tau_{0}-\tau \sim 0$ on this set. It follows that $P_{i}$ must be finite.

REMARK 3. The "dual" of Theorem 2 is false. There exists a set of types $T=\left\{\tau_{i} \mid 1 \leqslant i<\infty\right\}$ and a type $\tau_{0}$ such that $\tau_{0}=\tau_{i} \wedge \tau_{j}$ for all $i \neq j$, but $T$ is not the typeset of a rank two group [5, Example 1].

\section{REFERENCES}

1. D. Arnold, B. O'Brien and J. Reid, Quasi-pure injective and projective torsion free abelian groups of finite rank, Proc. London Math. Soc. (3) 38 (1979), 532-544.

2. D. Arnold, C. Vinsonhaler and W. Wickless, Quasi-pure projective and injective torsion free abelian groups of rank two, Rocky Mountain J. Math. 6 (1976), 61-70.

3. R. Beaumont and R. Pierce, Torsion-free groups of rank two, Mem. Amer. Math. Soc., no. 38, (1961).

4. R. Beaumont and R. Wisner, Rings with additive group which is a torsion-free group of rank two, Pacific J. Math. 1 (1951), 169-177.

5. D. Dubois, Applications of analytic number theory to the study of type sets of torsion-free abelian groups I and II, Publ. Math. Debrecen 12 (1965), 59-63 and 13 (1966), 1-18. 
6. L. Fuchs, Infinite abelian groups, Vol. I and II, Academic Press, New York, 1970 and 1973.

7. R. Ito, On type-sets of torsion-free abelian groups of rank two, Proc. Amer. Math. Soc. 48 (1975), $39-42$.

8. J. Koehler, Some torsion free rank two groups, Acta Sci. Math. (Szeged) 25 (1964), 186-190.

9. $\quad$ The type set of a torsion free group of finite rank, Illinois J. Math. $9(1965), 66-86$.

10. P. Schultz, The typeset and cotypeset of a rank two abelian group, Pacific J. Math. 78 (1978), 503-517.

11. __ Torsion-free extensions of torsion-free abelian groups, J. Algebra 30 (1974), 75-91.

12. C. Vinsonhaler and W. Wickless, Projective classes of torsion-free abelian groups (to appear).

13. R. Warfield, Homomorphisms and duality of torsion-free groups, Math. Z. 107 (1968), 189-200.

Department of Mathematics, University of ConNecticut, Storrs, ConNecticut 06268 